ARTICLE

Received 4 May 2015 | Accepted 12 Nov 2015 | Published 15 Dec $2015 \quad$ DOl: 10.1038/ncomms10185 OPEN

\title{
SCF $\beta$-TRCP promotes cell growth by targeting PR-Set7/Set8 for degradation
}

Zhiwei Wang ${ }^{1,2, \star}$, Xiangpeng Dai ${ }^{2, \star}$, Jiateng Zhong ${ }^{2,3, \star}$, Hiroyuki Inuzuka ${ }^{2}$, Lixin Wan ${ }^{2}$, Xiaoning Li ${ }^{2,4}$, Lixia Wang ${ }^{1}$, Xiantao $\mathrm{Ye}^{1}$, Liankun Sun ${ }^{4}$, Daming Gao ${ }^{2,5}$, Lee Zou ${ }^{6} \&$ Wenyi Wei ${ }^{2}$

The Set8/PR-Set7/KMT5a methyltransferase plays critical roles in governing transcriptional regulation, cell cycle progression and tumorigenesis. Although CRL4 ${ }^{\mathrm{Cdt} 2}$ was reported to regulate Set8 stability, deleting the PIP motif only led to partial resistance to ultravioletinduced degradation of Set8, indicating the existence of additional E3 ligase(s) controlling Set8 stability. Furthermore, it remains largely undefined how DNA damage-induced kinase cascades trigger the timely destruction of Set8 to govern tumorigenesis. Here, we report that SCF-TRCP earmarks Set8 for ubiquitination and degradation in a casein kinase I-dependent manner, which is activated by DNA-damaging agents. Biologically, both CRL4 ${ }^{\mathrm{Cdt} 2}$ and $\mathrm{SCF}^{\beta}$-TRCP -mediated pathways contribute to ultraviolet-induced Set8 degradation to control cell cycle progression, governing the onset of DNA damage-induced checkpoints. Therefore, like many critical cell cycle regulators including p21 and Cdt1, we uncover a tight regulatory network to accurately control Set8 abundance. Our studies further suggest that aberrancies in this delicate degradation pathway might contribute to aberrant elevation of Set8 in human tumours.

\footnotetext{
${ }^{1}$ The Cyrus Tang Hematology Center and Collaborative Innovation Center of Hematology, Jiangsu Institute of Hematology, the First Affiliated Hospital, Soochow University, Suzhou 215123, P.R. China. ${ }^{2}$ Department of Pathology, Beth Israel Deaconess Medical Center, Harvard Medical School, Boston, Massachusetts 02215, USA. ${ }^{3}$ Department of Pathology, Xinxiang Medical University, Xinxiang 453000, P.R. China. ${ }^{4}$ Department of Pathophysiology, Basic Medical College, Jilin University, Changchun 130021, P.R. China. ${ }^{5}$ State Key Laboratory of Cell Biology, Institute of Biochemistry and Cell Biology, Shanghai Institutes for Biological Sciences, Chinese Academy of Sciences, 320 Yue-yang Road, Shanghai 200031, P.R. China. ${ }^{6}$ Department of Pathology,

Massachusetts General Hospital Cancer Center, Harvard Medical School, Boston, Massachusetts 02129, USA. * These authors contributed equally to this work. Correspondence and requests for materials should be addressed to Z.W. (email: zwwang@suda.edu.cn) or to W.W. (email:

wwei2@bidmc.harvard.edu).
} 
$\mathrm{P}$ ost-translational modifications of histones play a critical role in a number of cellular processes such as mitosis, meiosis and the DNA damage response ${ }^{1}$. These modifications include methylation, acetylation, phosphorylation and ubiquitination, which often occur on the N-terminal tails of histone proteins. Importantly, emerging evidence has suggested that histone methylation is one of the important posttranslational modifications with pivotal biological consequences. Specifically, it has been revealed that histone H4 Lys 20 (H4K20) is one of the methylated lysine residues on the $\mathrm{H} 4 \mathrm{~N}$-terminal tail. In mammals, three methyltransferases including Set8, Suv4-20h1 and Suv4-20h2 have been identified to regulate the H4K20 methylation (mono-, di- and tri-methylation) status ${ }^{1}$. Among these methyltransferases, Suv4-20h1/h2 promotes the transition from H4K20me1 to di- and tri-methylation of H4K20 $(\mathrm{H} 4 \mathrm{~K} 20 \mathrm{me} / 3)^{2}$. On the other hand, the Set8/PR-Set7/lysine methyltransferase 5a (KMT5a), a sole monomethyltransferase, primarily regulates the monomethylation of H4K20 (refs 3,4). Knockout mouse studies have further revealed that Set8 is required for developmental processes and loss of Set8 could cause cell cycle defects and increased DNA damage ${ }^{3,4}$. Besides H4K20, Set8 has also been found to methylate non-histone proteins including the p53 tumour-suppressor protein, subsequently preventing p53 from promoter binding to inhibit the transcriptional activation of $\mathrm{p} 21$ and $\mathrm{p} 53$ upregulated modulator of apoptosis (PUMA) ${ }^{5}$.

Several lines of evidence have defined that Set8 exerts its biological functions in regulating cell cycle and DNA damage response in part through its interaction with a number of nuclear proteins, such as proliferating cell nuclear antigen (PCNA), RNA polymerase II, ER, LEF3 and TWIST ${ }^{1}$. As Set8 plays an important role in various cellular processes, its activity needs to be tightly regulated for the precise control of cell fate. To this end, a wealth of evidence has revealed that Set 8 could be regulated at both the transcriptional level ${ }^{6}$ and by post-translational modification $(\mathrm{s})^{1}$. Multiple enzymes such as kinases, small ubiquitin-like modifier, and ubiquitin ligases have been reported to control Set8 modification. For example, Cyclin B/Cdk1 phosphorylates Set8 at Ser29 during mitosis ${ }^{7}$, and the E3 ubiquitin ligase complex, CRL4 ${ }^{\mathrm{Cdt} 2}$, governs ubiquitination-mediated Set 8 degradation ${ }^{4,8,9}$. In addition, the E3 ubiquitin ligases $\mathrm{SCF}^{\mathrm{Skp} 2}$ and B-lymphoma and BAL-associated protein have also been reported to regulate Set8 stability ${ }^{10,11}$, but no physiological evidence has been obtained to support whether B-lymphoma and BAL-associated protein or SCF ${ }^{\mathrm{Sk} 2}$ directly ubiquitinates Set8. Moreover, the anaphase-promoting complex APC ${ }^{\mathrm{Cdh} 1}$ has also recently been found to promote the ubiquitination of Set 8 and subsequent proteolysis ${ }^{7}$. However, although Set8 destruction has been reported to be stimulated by ultraviolet $^{12}$, it remains largely unclear whether DNA damage-induced kinase cascades play a critical role in this process. In the present study, we report that Set8 is an ubiquitin substrate of $\beta$-TRCP ( $\beta$-transducin repeatcontaining protein), and Set8 ubiquitination and subsequent degradation is timely governed by the E3 ubiquitin ligase $\mathrm{SCF}^{\beta-T R C P}$ in a casein kinase I (CKI)-dependent manner.

$\beta$-TRCP is one of the $69 \mathrm{~F}$-box proteins that form the SCF (Skp1-Cullin-1-F-box protein) type of E3 ligase complexes. Notably, the SCF complex is composed of Skp1, Cullin-1, Ring box protein-1 (Rbx1)/Roc1 and one of the 69 F-box proteins. SCF ${ }^{\beta-T R C P}$ often targets substrates containing the consensus sequence DSGXXS degron ${ }^{13}$. Moreover, SCF $^{\beta-T R C P}$-mediated ubiquitination and degradation requires specific kinases to phosphorylate two serine residues within the phosphodegron of its substrates ${ }^{13}$. A growing list of SCF ${ }^{\beta-T R C P}$ ubiquitin substrates have recently been identified including EMI-1 (early mitotic inhibitor-1) ${ }^{14,15}$, Wee1 (ref. 16), and Cdc25A (cell division cycle 25 homologue $A)^{17,18}$. Biologically, these substrates regulate cell cycle and cellular apoptosis, indicating that $\beta$-TRCP is critically a

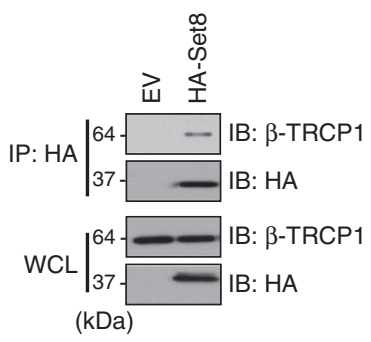

e

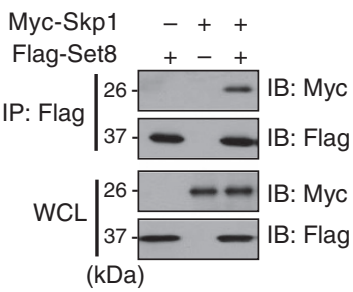

b

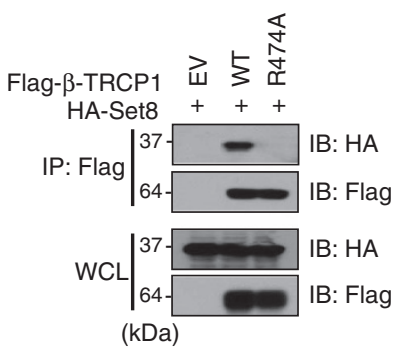

$\mathbf{f}$

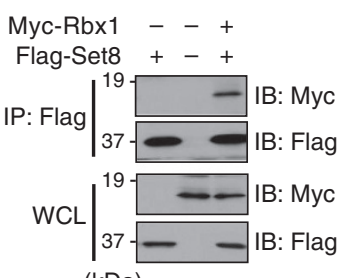

C

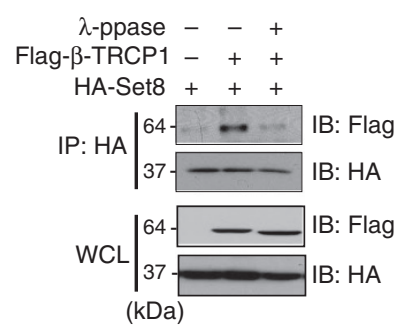

g

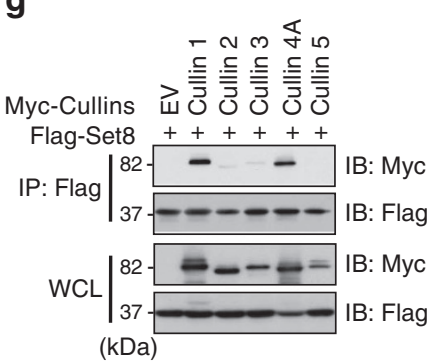

d

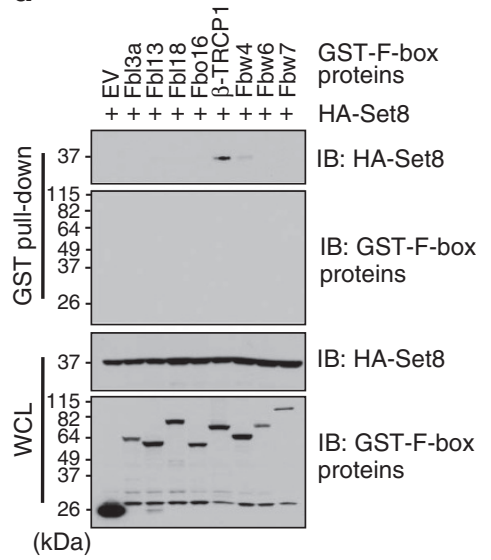

Figure 1 | Set8 interacts with the SCF ${ }^{\boldsymbol{\beta} \text {-TRCP }}$ complex. (a) Immunoblot (IB) analysis of whole cell lysates (WCL) and immunoprecipitates (IP) derived from HeLa cells transfected with HA-Set8 or empty vector (EV) as a negative control. (b) IB analysis of WCL and IP derived from HeLa cells transfected with HA-Set8 and Flag-tagged wild type or R474A mutant $\beta$-TRCP1 constructs, or EV as a negative control. (c) IB analysis of WCL and IP derived from HeLa cells transfected with HA-Set8 and Flag- $\beta$-TRCP1 constructs as indicated. Where indicated, cell lysates were pre-treated with $\lambda$-phosphatase before the IP procedure. (d) IB analysis of WCL and GST pull-down derived from HeLa cells transfected with HA-Set8 and the indicated GST-F-box proteins. (e) IB analysis of WCL and IP derived from HeLa cells transfected with Flag-Set8 and Myc-Skp1 constructs, as indicated. (f) IB analysis of WCL and IP derived from HeLa cells transfected with Flag-Set8 and Myc-Rbx1 constructs, as indicated. (g) IB analysis of WCL and IP derived from HeLa cells transfected with Flag-Set8 and Myc-Cullin1, 2, 3, 4A or 5 expression plasmids. 
involved in governing proper cell cycle progression and cell survival $^{13}$. Here, we report that Set8 interacts with the SCF ${ }^{\beta-T R C P}$ complex and depletion of endogenous $\beta$-TRCP leads to an accumulation of the Set8 protein. Moreover, our results reveal a critical role of the CKI kinase in $\mathrm{SCF}^{\beta-\mathrm{TRCP}}$-mediated degradation of Set8. Furthermore, inhibition of CKI-mediated phosphorylation of Set8 at Ser253 suppresses its destruction by SCF ${ }^{\beta \text {-TRCP }}$. More importantly, $\beta$-TRCP-mediated degradation of Set8 affects cell growth and cell cycle progression. Hence, our current study supports a pivotal role of $\beta$-TRCP in CKI-mediated Set8 degradation, and further implies that targeting $\beta$-TRCP could be a novel approach to govern cell cycle progression in part by regulating Set8 destruction.

\section{Results}

Set8 interacts with $\mathrm{SCF}^{\beta-\mathrm{TRCP}} \mathrm{E} 3$ ubiquitin ligase complex. To better understand the critical role of the Set8 methyltransferase in carcinogenesis, we employed an affinity purification coupled with the mass spectrometry approach to identify proteins associated with Set8 (refs 19-21). Our mass spectrometry results showed that Set8 co-purified with multiple proteins including PCNA $^{4,22}$ and $\beta$-TRCP (Supplementary Fig. 1a). To validate $\beta$-TRCP as a novel Set8-interacting protein, we performed coimmunoprecipitation experiments and observed that exogenously expressed Set 8 associated with endogenous $\beta$-TRCP1 (Fig. 1a and Supplementary Fig. 1b,c). We further demonstrated that the interaction of $\beta$-TRCP1 and Set8 was significantly reduced using a $\beta$-TRCP1 mutant (R474A) harbouring a point mutation within the substrate interaction site of $\beta$-TRCP1 (ref. 13; Fig. 1b and Supplementary Fig. 1d). It is well characterized that proper phosphorylation of substrates by kinases is required for SCF $^{\beta-T R C P}$-mediated ubiquitination and degradation ${ }^{13,23}$. In keeping with this notion, we found that the $\beta$-TRCP/Set8 interaction was largely abolished by the phosphatase treatment (Fig. 1c). More importantly, using a panel of F-box proteins, we observed that Set8 specifically bound $\beta$-TRCP1, but not other F-box proteins we examined (Fig. 1d), further suggesting for a specific interaction between Set8 and $\beta$-TRCP1. As additional supporting evidence, we identified Set8 to interact with numerous known SCF components including Skp1 (Fig. 1e) and Rbx1 (Fig. 1f), indicating that the $\mathrm{SCF}^{\beta}$-TRCP holo-complex may be involved in governing Set8 stability. Moreover, in addition to the reported interaction between Set8 and Cullin 4A (refs 4,8,9), we observed that Set8 also interacted with Cullin 1, but not other Cullin family members in cells (Fig. $1 \mathrm{~g}$ and Supplementary Fig. 1e).

Set8 stability is negatively controlled by $\beta$-TRCP. To determine whether Set8 is a bone fide substrate of SCF ${ }^{\beta-T R C P}$, we next assessed Set 8 protein abundance changes in cells after depleting endogenous $\beta$-TRCP. Notably, we found that depletion of endogenous $\beta$-TRCP1, but not other F-box proteins we examined, led to an elevation in Set8 protein levels (Fig. 2a-c and Supplementary Fig. 2a-c). In keeping with the notion that both Cullin 1 and Cullin 4 were involved in regulation of Set 8 stability, an increase in Set8 abundance was observed after depleting either endogenous Cullin 1 or endogenous Cullin $4 A$, but not Cullin $4 B$ (Fig. 2d and Supplementary Fig. 2d,e). However, we did not observe an additive elevation in the abundance of endogenous Set8 protein in Cullin $4 A$ and Cullin $4 B$ double knockdown cells compared with either protein depleted individually (Supplementary Fig. 2e), indicating that Cullin 4A, but not Cullin $4 \mathrm{~B}$ plays a critically important physiological role in the regulation of Set8 degradation in cells. In further support of this notion, we observed that Cullin 4A, but not Cullin 4B interacts with Set8 in cells (Supplementary Fig. 2f,g). As such, there are elevated Set8 protein levels upon genetic deletion of Culin $4 A$, but not Cullin $4 B$ in mouse embryonic fibroblasts (Supplementary Fig. 2h,i).

Moreover, treatment with the $26 \mathrm{~S}$ proteasome inhibitor MG132 caused an upregulation of Set8 protein levels, indicating the involvement of the $26 \mathrm{~S}$ proteasome in governing Set 8 stability (Fig. 2e and Supplementary Fig. 2j). Furthermore, compared with control cells, depletion of endogenous $\beta$-TRCP1 led to an elevation of Set 8 protein abundance in the G1 phase (Fig. $2 \mathrm{f}$ and Supplementary Fig. 2k), largely through extending the half-life of endogenous Set8 protein (Fig. 2g,h and Supplementary Fig. 2l), indicating that $\beta$-TRCP primarily governs Set8 abundance through a post-transcriptional mechanism.

CKI is involved in the regulation of Set8 stability. Emerging evidence has demonstrated that one kinase or a combination of multiple kinases are required to properly phosphorylate substrates within their phosphodegron before substrate ubiquitination and degradation by the SCF type of E3 ligases including $\mathrm{SCF}^{\beta-\mathrm{TRCP}}$ (refs 13,23). To this end, it has been previously reported that $\mathrm{CKI}$ is often involved in $\beta$-TRCP-mediated protein degradation. Therefore, we sought to explore whether CKI is the upstream kinase that phosphorylates Set8 and subsequently triggering its destruction by SCF ${ }^{\beta-T R C P}$. Notably, we observed that both CKI $\delta$ and CKI $\alpha$ specifically interacted with Set8 (Fig. 3a). Moreover, depletion of endogenous $C K I \delta$, and to a lesser extent, depletion of $C K I \alpha$, but not $C K I \varepsilon$, led to an accumulation of Set8 (Fig. 3b). Conversely, ectopic expression of either $\mathrm{CKI} \delta$ or $\mathrm{CKI} \alpha$ promoted the destruction of ectopically expressed Set8 (Fig. 3c). Consistently, degradation of Set8 was blocked after MG132 treatment (Fig. 3c), suggesting that the 26S proteasome is involved in this process. In further support of a physiological role for CKI in Set8 stability control, inactivating CKI by either depletion of CKI $\delta$ by shRNA or using a CKI inhibitor, D4476, significantly disrupted the interaction between Set8 and $\beta$-TRCP1 (Fig. 3d,e). Consistently, pharmacological inactivation of CKI by D4476 or IC261 led to an elevation of Set8 abundance in a dose-dependent manner (Fig. $3 \mathrm{f}$ and Supplementary Fig. 3a-d). More importantly, we demonstrated

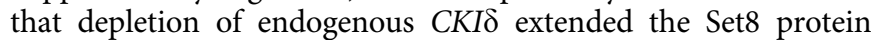
half-life (Fig. 3g,h), whereas conversely, ectopic expression of CKI $\delta$ led to a reduction in the half-life of endogenous Set8 (Supplementary Fig. 3e,f). These results coherently support the notion that CKI is critically involved in the destruction of Set8 by $\mathrm{SCF}^{\beta-\mathrm{TRCP}}$.

CKI $\delta$-mediated phosphorylation of Set8 at the Ser253 site. It has been reported by multiple groups that most $\beta$-TRCP substrates contain the canonical D/ESGxxS degron sequence ${ }^{13,21,24}$. Indeed, we observed that Set8 contains a putative ESGxxE degron that is conserved among various species (Fig. 4a). To further determine whether this putative phosphodegron is important for Set8 degradation, we mutated the Ser253 residue to alanine (thereafter termed the S253A mutant). Notably, in vivo degradation assays revealed that ectopic expression of $\beta$-TRCP1 and CKI $\delta$ led to a rapid disappearance of wild-type (WT), but not the S253A mutant form of, Set8 that is deficient in associating with $\beta$-TRCP1 (Fig. 4b,c and Supplementary Fig. 4a,b). Notably, MG132 prevented the degradation of Set8 (Fig. $4 \mathrm{~b}$ and Supplementary Fig. 4a), indicating the involvement of the $26 \mathrm{~S}$ proteasome pathway in mediating Set8 degradation by

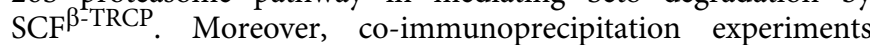
demonstrated that $\beta$-TRCP1 bound the middle domain of Set8 that contains the identified phosphodegron motif (Fig. $4 \mathrm{~d}, \mathrm{e}$ ), further supporting a critical role for Ser253 in mediating 
a

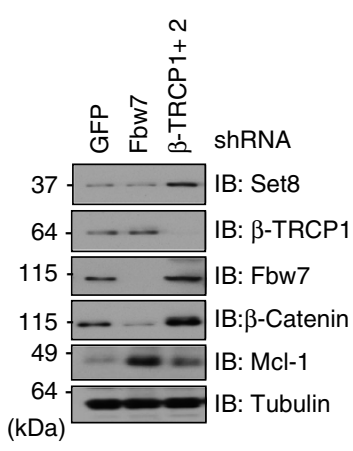

b

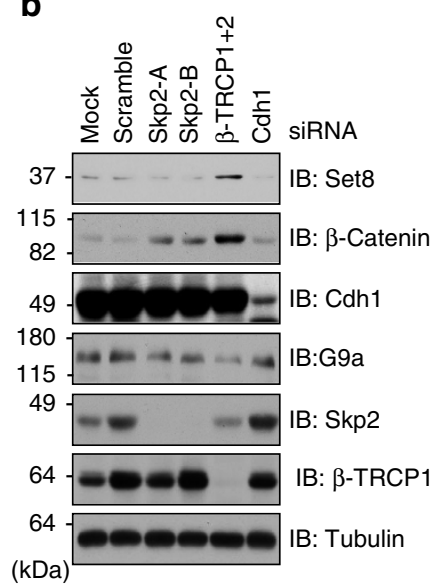

C

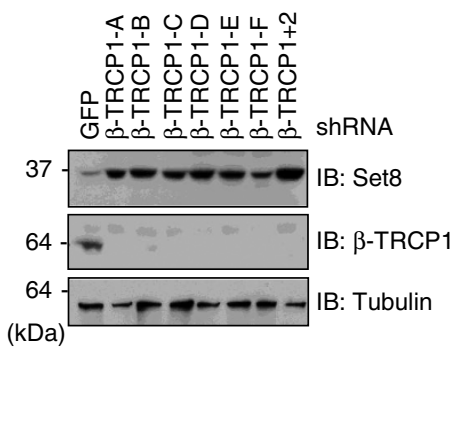

d e

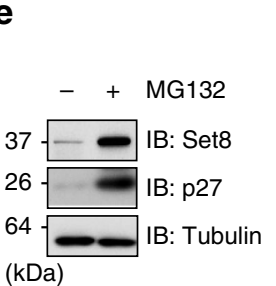

\section{f}

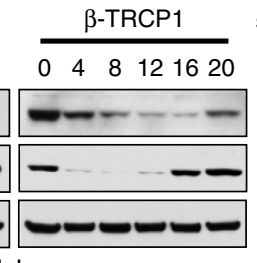

$\mathrm{HeLa}$ g

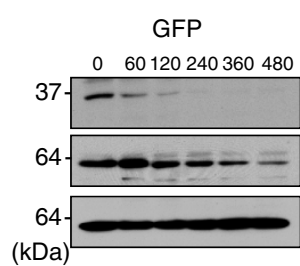

$\beta$-TRCP1

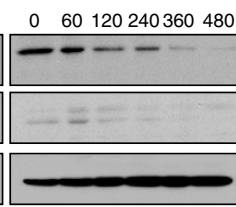

$\beta-T R C P 1+2$ o 60120240360480

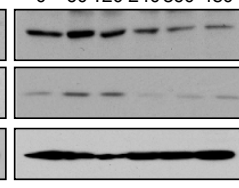

ShRNA

$\mathrm{CHX}$ (min)

IB: Set8

IB: $\beta$-TRCP1

IB: Tubulin
shRNA
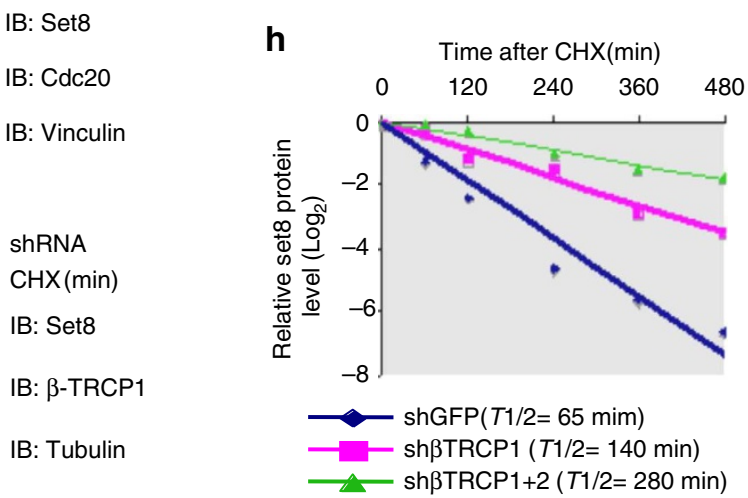

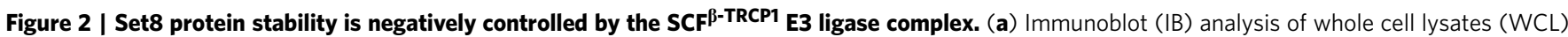
derived from HeLa cells infected with the indicated shRNA constructs. (b) IB analysis of HeLa cells transfected with the indicated siRNA oligonucleotides. (c) IB analysis of WCL derived from HeLa cells infected with shRNA constructs specific for GFP, $\beta$-TRCP1 (six independent lentiviral $\beta$-TRCP1-targeting shRNA constructs namely, $-A,-B,-C,-D,-E,-F)$, or $\beta-T R C P 1+2$, followed by selection with $1 \mu \mathrm{g} \mathrm{ml}-1$ puromycin for 3 days to eliminate non-infected cells. (d) IB analysis of WCL from 293 T cells infected with shRNA specific for GFP, or several shRNA constructs against Cullin 1 (three independent lentiviral Cullin 1-targeting shRNA constructs namely, $-A,-B,-C)$ followed by selection with $1 \mu \mathrm{g} \mathrm{ml}{ }^{-1}$ puromycin for 3 days to eliminate the non-infected cells. (e) IB analysis of WCL derived from 293 T cells with or without MG132 treatment. (f) HeLa cells were infected with the shGFP or sh $\beta$-TRCP1 followed by selection with $1 \mu \mathrm{g} \mathrm{ml}^{-1}$ puromycin for 3 days to eliminate non-infected cells. The generated stable cell lines were then treated with nocodazole to arrest at the $\mathrm{M}$ phase, and then release back to the cell cycle by washing off nocodazole. At the indicated time points, WCL were prepared and immunoblots were probed with the indicated antibodies. (g) HeLa cells were infected with the shRNA constructs for GFP, $\beta$-TRCP1 or $\beta$-TRCP1 2 followed by selection with $1 \mu \mathrm{g} \mathrm{ml}^{-1}$ puromycin for 3 days to eliminate non-infected cells. The generated stable cell lines were then split into 60 -mm dishes. After $20 \mathrm{~h}$, cells were treated with $20 \mu \mathrm{g} \mathrm{ml}^{-1}$ cycloheximide $(\mathrm{CHX})$. At the indicated time points, WCL were prepared and immunoblots were probed with the indicated antibodies. (h) Quantification of the Set8 band intensities in $\mathbf{g}$. Set8 band intensity was normalized to tubulin, and then normalized to the $t=0$ controls.

$\beta$-TRCP1 interaction with Set8 in a phosphorylation-dependent manner (Fig. 1c).

Interestingly, the Set $8^{\Delta \mathrm{PIP}}$ mutant that lacks the $\mathrm{CRL} 4^{\mathrm{Cdt} 2}$ recognizable degron motif, thereby deficient in associating with Cullin 4A (ref. 9), could still be efficiently degraded by co-expression of CKI $\delta$ and $\beta$-TRCP (Fig. $4 \mathrm{f}$ ), a process that can be blocked by MG132 treatment (Fig. 4g). Furthermore, unlike Set $8^{\mathrm{WT}}$ and Set $8^{\Delta \mathrm{PIP}}$, Set $8^{\text {S253A }}$ and Set $8^{\Delta \mathrm{PIP} / \mathrm{S} 253 \mathrm{~A}}$ were deficient in binding $\beta$-TRCP1 (Fig. $4 \mathrm{~h}$ and Supplementary Fig. 4c), supporting the notion that $\mathrm{SCF}^{\beta-\mathrm{TRCP}}$ regulates Set8 stability independent of CRL4 ${ }^{\text {Cdt } 2}$ and the PIP motif. Moreover, point mutation of the $\beta$-TRCP recognition motif of Set8, namely the Ser253 residue, but not the PIP motif, abolished the association between Set8 and Cullin 1 (Fig. 4i), as well as the interaction between Set8 and other SCF components such as Rbx1 (Supplementary Fig. 4d) and Skp1 (Supplementary Fig. 4e). More importantly, we showed that $\beta$-TRCP1 promoted the in vitro ubiquitination of WT-, but not the S253A mutant form of, Set8, in a CKI $\delta$-dependent manner (Supplementary Fig. 4f). This result further suggests that the inefficient destruction of S253A by SCF $^{\beta-T R C P}$ (Fig. 4b) could be likely due to deficient ubiquitination of Set8. Notably, ultraviolet irradiation augmented the interaction between Set 8 and $\beta$-TRCP1, which was impeded by the treatment of CKI inhibitor D4476 (Fig. 4j), supporting a critical role for both SCF ${ }^{\beta-T R C P}$ and CKI in ultraviolet-induced degradation of Set8. Consistently, in vivo ubiquitination assays further demonstrated that SCF ${ }^{\beta-T R C P}$ promoted Set8 poly-ubiquitination in a Ser253-dependent manner (Fig. 4k and Supplementary Fig. 4g). Importantly, using mass spectrometry analysis, we identified Ser253 phosphorylation in cells (Supplementary Fig. 4h). Furthermore, the Ser253 residue is predicted to be a putative CKI site, which prefers $\mathrm{D} / \mathrm{ExxS}$ or $\mathrm{D} / \mathrm{ExxxS}$ consensus sequence 21,24 . Taken together, these findings suggest that CKI $\delta$ could phosphorylate Set8 at S253 to trigger Set8 destruction. 
a

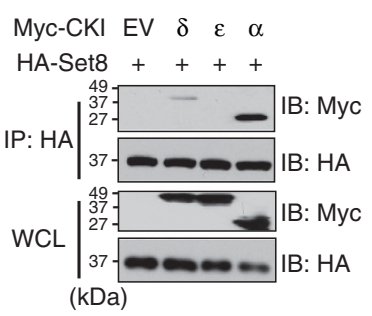

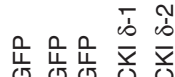
ShRNA 0 OO 0 O O HA-Set8 -++++ Flag- $\beta$-TRCP1 ++++

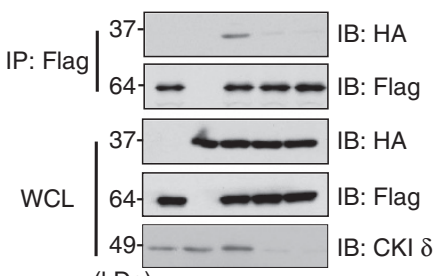
$(\mathrm{kDa})$

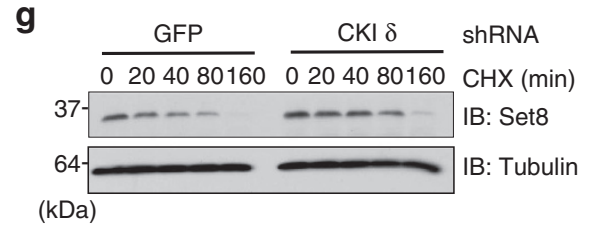

b

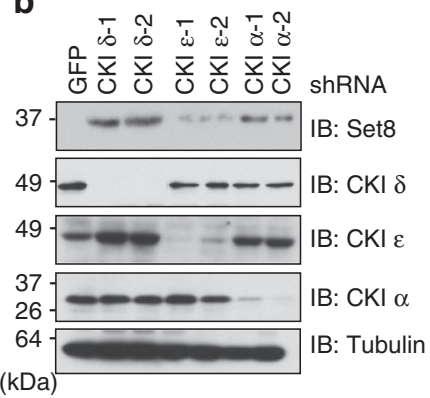

e

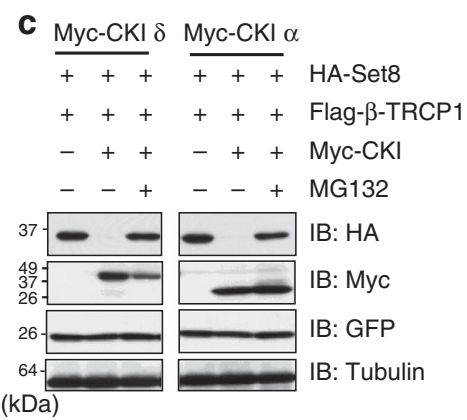

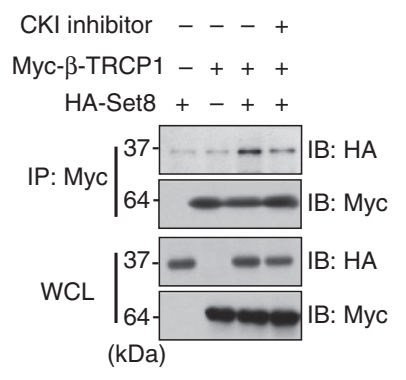

h

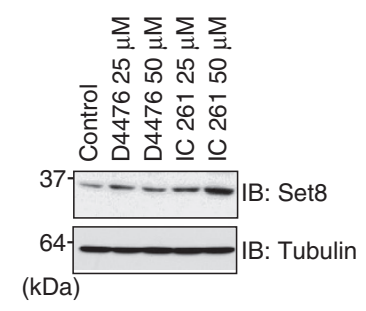

$(\mathrm{kDa})$

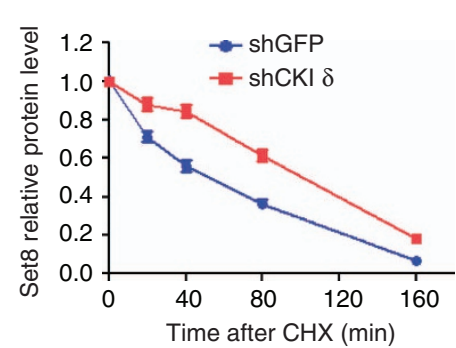

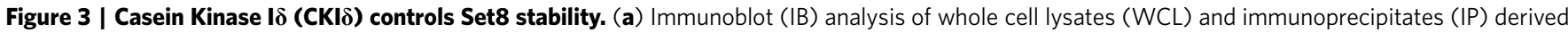
from HeLa cells transfected with HA-Set8 and Myc-tagged versions of the indicated CKI isoforms. (b) IB analysis of HeLa cells that were infected with shRNA specific for GFP or the indicated CKI isoforms (two independent shRNAs, namely -1 and -2 ), followed by selection with $1 \mu \mathrm{g} \mathrm{ml}{ }^{-1}$ puromycin for 3 days to eliminate non-infected cells. (c) IB analysis of WCL derived from HeLa cells transfected with HA-Set8, Flag- $\beta$-TRCP and indicated kinases. Where indicated, cells were treated with MG132. (d) IB analysis of WCL and IP derived from HeLa cells infected with shGFP or shCKI $\delta$ followed by selection with $1 \mu \mathrm{g} \mathrm{ml}^{-1}$ puromycin for 3 days to eliminate non-infected cells. (e) IB analysis of WCL and IP derived from HeLa cells transfected with HA-Set8 and Myc- $\beta$-TRCP1. Where indicated, cells were treated with the CKI inhibitor D4476 for $10 \mathrm{~h}$ before collection. (f) IB analysis of HeLa cells treated with the CKI inhibitor D4476 and IC261 at the indicated concentrations for $12 \mathrm{~h}$. (g) HeLa cells were infected with the shRNA constructs for GFP, or CKI $\delta$ followed by selection with $1 \mu \mathrm{g} \mathrm{ml}^{-1}$ puromycin for 3 days to eliminate non-infected cells. The generated stable cell lines were then split into 60 -mm dishes. Cells were treated with $20 \mu \mathrm{g} \mathrm{ml}^{-1} \mathrm{CHX}$ after $20 \mathrm{~h}$. At the indicated time points, WCL were prepared and immunoblots were probed with the indicated antibodies. (h) Quantification of the Set8 band intensities in $\mathbf{g}$. Set8 band intensity was normalized to tubulin, and then normalized to the $t=0$ controls.

Effects of $\beta$-TRCP-mediated Set8 degradation on cell growth. It has been previously reported that Set8 protein levels were significantly reduced after DNA damage induced by ultraviolet C (UVC) treatment ${ }^{8,9}$. Consistent with these findings, we found that UVC could promote the degradation of ectopically expressed WT-Set8 in a dose-dependent manner (Fig. 5a). In support of a critical role of $\beta$-TRCP in UVC-induced degradation of Set8, we found that mutating the critical Ser253 residue within the $\beta$-TRCP recognizable degron motif, or depleting endogenous $\beta$-TRCP1, largely abolished ultraviolet-induced reduction of Set8 abundance, in part due to extended Set8 protein half-life (Fig. 5a-c and Supplementary Fig. 5a-c). However, we still observed a reduction of S253A-Set8 abundance on high-dose UVC treatment (25 and $50 \mathrm{~J} \mathrm{~m}^{-2}$; Fig. 5a,b).

Importantly, previous studies have also shown that ultravioletinduced Set8 degradation is in part dependent on its PIP degron ${ }^{9}$. In line with this concept, we found that the Set ${ }^{\Delta \mathrm{PIP}}$ mutant is more stable than Set $8^{\mathrm{WT}}$ after UVC treatment (Fig. 5a,b and
Supplementary Fig. 5b,c). However, the abundance of the Set $8^{\triangle \mathrm{PIP}}$ mutant is also reduced in relatively high-dose UVC treatment (25 and $50 \mathrm{~J} \mathrm{~m}^{-2}$; Fig. $\left.5 \mathrm{a}, \mathrm{b}\right)$. On the other hand, simultaneous inactivation of both degrons created a mutant version of Set8 that are more resistant to UVC-induced degradation, even in high dosage of UVC treatment (Fig. 5a,b). These data suggest the possibility that both Cdt2 and $\beta$-TRCP have non-redundant roles in governing UVC-induced Set8 ubiquitination and degradation, such that simultaneous inactivation of both CRL4 ${ }^{\mathrm{Cdt}}{ }_{-}$and $\mathrm{SCF}^{\beta-T R C P}$-mediated pathways are required to abolish UVC-induced degradation of Set8 in cells.

Furthermore, given its critical role in epigenetic and cell cycle regulation, previous studies have revealed that forced Set8 expression slowed down cell proliferation ${ }^{9}$. Consistent with this report, we found that ectopic expression of Set $8^{\mathrm{WT}}$ inhibited cell proliferation. Moreover, ectopic expression of the relatively more stable version of the Set $8^{\Delta \mathrm{PIP}}$ and Set $8^{\mathrm{S} 253 \mathrm{~A}}$ mutants caused a 
a

Human: 247-IDELIESGKEEGMKIDLID-265

Bovine: 206-IDELIESGKEEGMKIDLID-224

Mouse: 204-INELIESGKEEGMKIDLID-221

Chicken: 180 -IDELITSGKEEGMKIDYID-198 $\beta$-TRCP D/ESGXXS

Degron motif

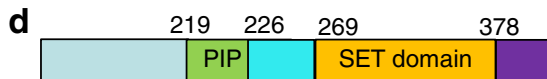

$\mathrm{N} 63 \longrightarrow 172$

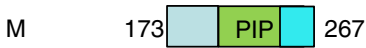

C $\quad 268$\begin{tabular}{|l|l|}
\hline SET domain & \\
\hline
\end{tabular}

e $\quad$ EV WT C M N HA-Set8

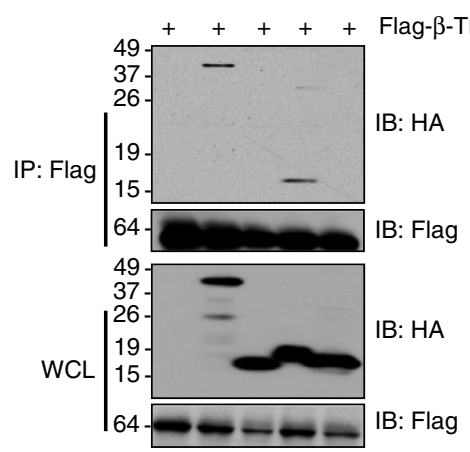

(kDa)

h
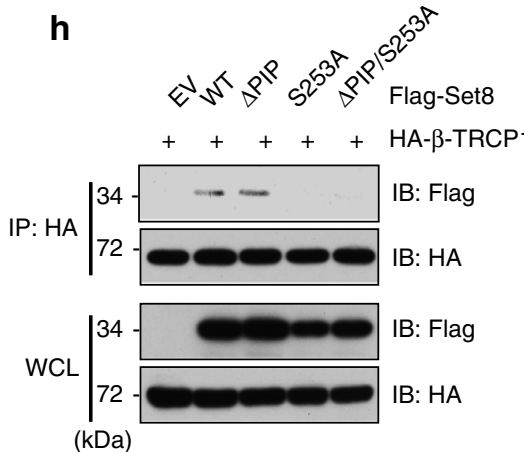

$(\mathrm{kDa})$

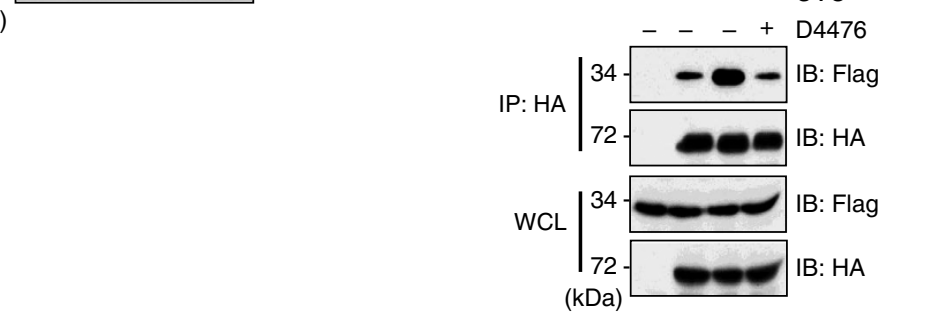

b

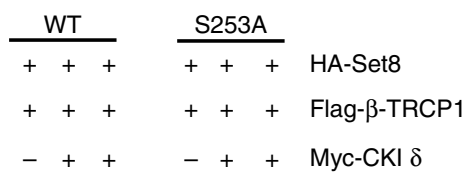

$-\ldots+\ldots+$ MG132

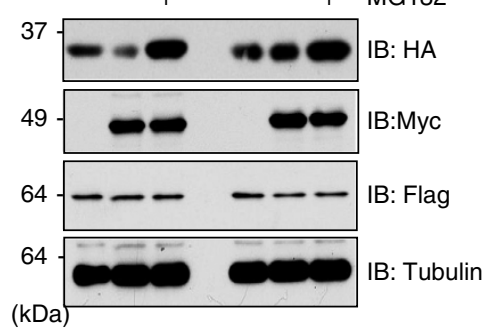

f

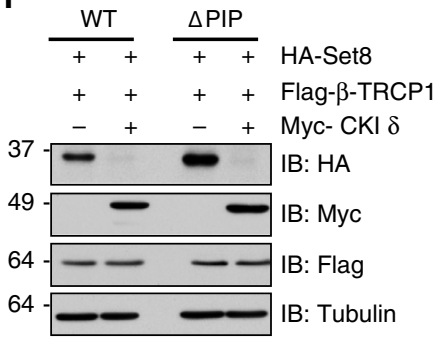

$(\mathrm{kDa})$

i
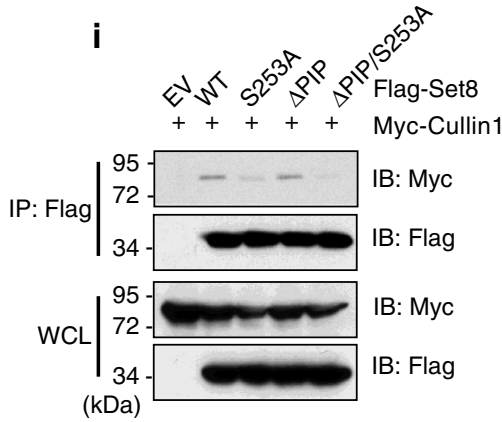

$\mathbf{j}$

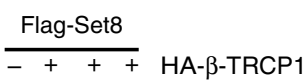

$\overline{-+++}$ HA- $\beta-$ TRCP1

- ++ + UVC

- $-\quad+$ D4476

$(\mathrm{kDa})$
C

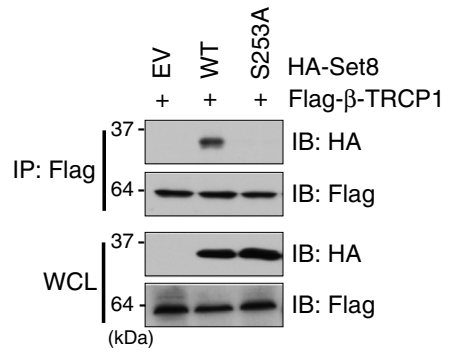

g

Flag-Set8- $\triangle$ PIP

-+++ HA- $\beta$-TRCP 1

-+++ Myc-CKI $\delta$

- _ _ + MG132

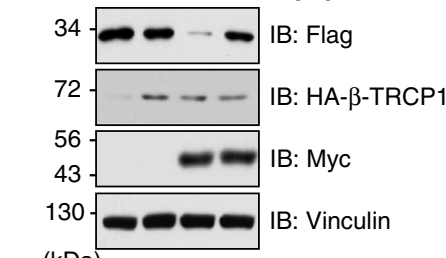

k

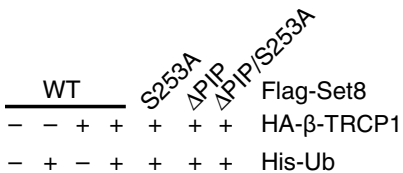

--+++++ Myc-CK $1 \delta$
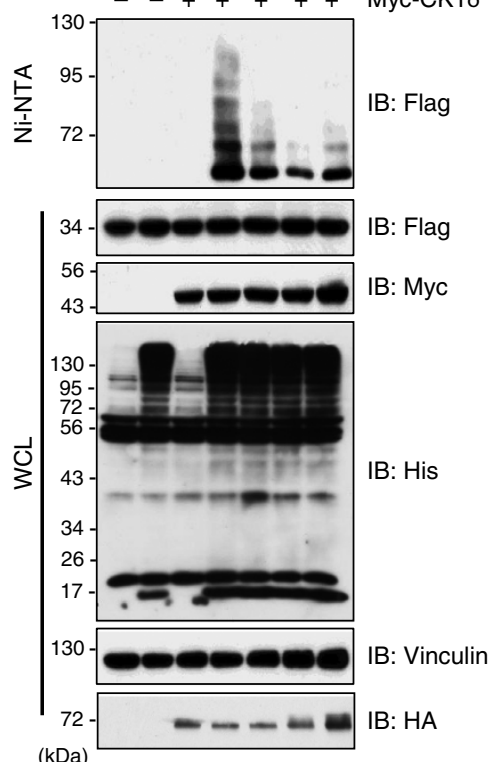

Figure 4 | CKI $\delta$ phosphorylates Set8 at S253 site. (a) Alignment of the candidate phosphodegron sequence in Set8 from different species.

(b) Immunoblot (IB) analysis of cells transfected with Flag- $\beta$-TRCP1 and HA-tagged wild type or the S253A mutant Set8 constructs, as indicated. Where indicated, cells were treated with Myc-CKIS, or the proteasome inhibitor MG132. (c) IB analysis of WCL and IP derived from HeLa cells transfected with Flag- $\beta$-TRCP1, HA-WT-Set8 and HA-S253A-Set8. (d) A schematic illustration of Set8 functional domains and the truncation mutants with different domains (C, M, N domain) that are used in e. (e) IB analysis of WCL and IP derived from HeLa cells transfected with Flag- $\beta$-TRCP1, HA-WT-Set8 and HA-Set8 constructs with different domains (C, M, N domain). (f) IB analysis of HeLa cells transfected with Flag- $\beta$-TRCP1, Myc-CKI $\delta$ and Flag-tagged wild-type or $\triangle \mathrm{PIP}$ mutant Set8 constructs, as indicated. (g) IB analysis of HeLa cells transfected with HA- $\beta$-TRCP1, Myc-CKI $\delta$ and Flag-tagged $\Delta$ PIP mutant Set8 constructs. Where indicated, $10 \mu \mathrm{M}$ MG132 was added for $12 \mathrm{~h}$ before collecting. (h) IB analysis of WCL and IP derived from HeLa cells transfected with HA- $\beta$-TRCP1 and indicated Flag-Set8 constructs. (i) IB analysis of WCL and IP derived from 293 T cells transfected with Flag-Set8 mutants and Myc-tagged Cullin1. (j) IB analysis of WCL and IP derived from 293 T cells transfected with Flag-Set8 and HA- $\beta$-TRCP1 and treated with D4476 under UVC exposure condition. (k) In vivo ubiquitination assays to demonstrate that SCF-TRCP promotes Set8 ubiquitination in a pSer253-dependent manner. His-pull-downed ubiquitinated Set8 and WCL were subjected to IB analysis with indicated antibodies. 
a

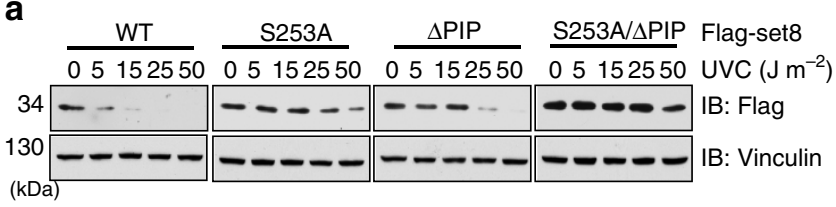

b

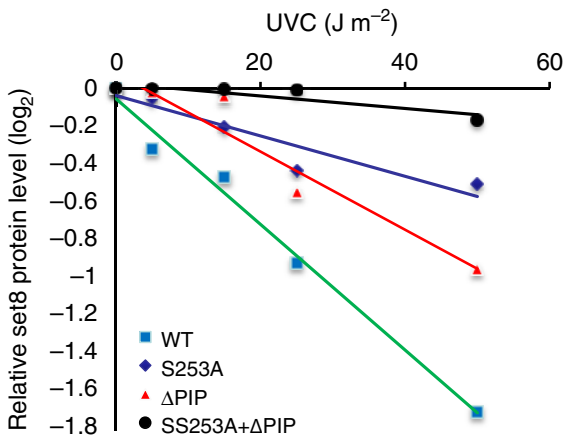

d

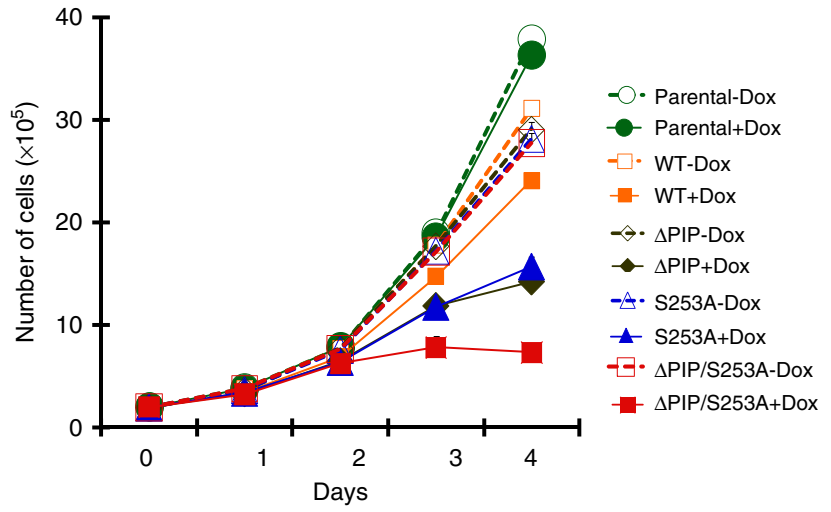

C

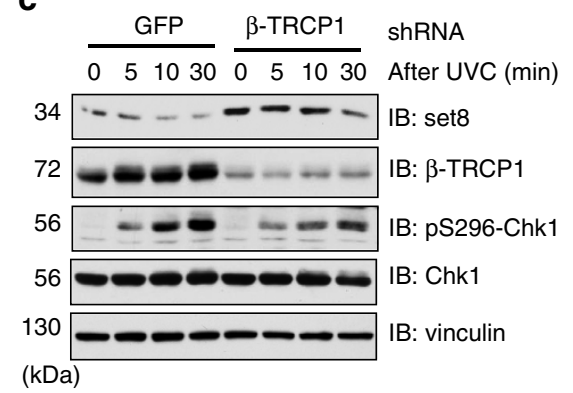

$\mathbf{e}$
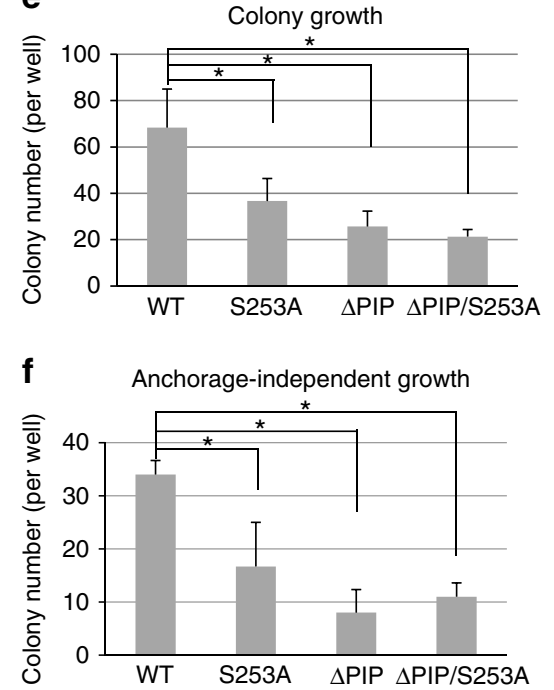

Figure 5 | Effects of $\boldsymbol{\beta}$-TRCP-mediated degradation of Set8 on cell growth. (a) U2OS cells with induced Flag-Set8 ${ }^{\text {WT }}$, Set $8^{\Delta P I P}$, Set $8^{\text {S253A }}$ or Set $8^{\Delta \mathrm{PIP} / \mathrm{S} 253 \mathrm{~A}}$ were treated with different doses of UVC $\left(0-50 \mathrm{~J} \mathrm{~m}^{-2}\right.$ ) for $10 \mathrm{~min}$ before whole cell lysates (WCL) were collected for immunoblot (IB) analysis to monitor the changes in Flag-Set8 abundance. (b) Quantification of the Flag-Set8 band intensities in a. Set8 band intensity was normalized to vinculin. (c) IB analysis of WCL from U2OS cells infected with lentivired shRNAs specific for GFP or $\beta$-TRCP1, treated with UVC (10 J m ${ }^{-2}$ ) and collected at indicated time points. (d-f) U2OS cells harbouring Flag-Set8WT, Set8 $8^{\triangle \mathrm{PIP}}$, Set8 $8^{\mathrm{S} 253 \mathrm{~A}}$ or Set8 ${ }^{\triangle \mathrm{PIP} / \mathrm{S} 253 \mathrm{~A}}$ were cultured in the absence or presence of $0.1 \mu \mathrm{g} \mathrm{ml}^{-1}$ doxycycline (Dox). The total cell numbers at the different days were plotted (d). Clonogenic assay (e) and soft agar assay (f) were used to measure cell proliferation. Data are shown as mean \pm s.d. of three independent experiments. ${ }^{\star} P<0.05$, Student's $t$-test.

more marked effect in inhibiting cell proliferation, compared with expression of Set 8 WT (Fig. 5d). More importantly, ectopic expression of the Set $8^{\Delta \mathrm{PIP} / 3253 \mathrm{~A}}$ mutant, which is much more resistant to UVC-induced degradation (Supplementary Fig. 5b,c), led to a greater inhibition of cell proliferation compared with the expression of Set $8^{\mathrm{WT}}$, Set $8^{\Delta \mathrm{PIP}}$ or Set $8^{\mathrm{S} 253 \mathrm{~A}}$ alone (Fig. $5 \mathrm{~d}$ and Supplementary Fig. 5d). Moreover, experimental results derived from colony formation assays further validated that Set $8^{\Delta \mathrm{PIP}}$ and Set $8^{\mathrm{S} 253 \mathrm{~A}}$ are more potent than Set $8 \mathrm{WT}$ in suppressing colony formation in vitro (Fig. 5e). Interestingly, disrupting both

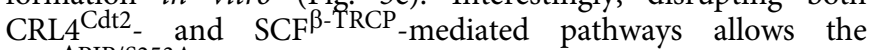
Set $8^{\Delta \text { PIP/S253A }}$ double mutant to be the most potent among all Set8 constructs we examined to inhibit colony growth, but not the anchorage-independent growth, in vitro (Fig. 5e,f). These results revealed both CRL4 ${ }^{\mathrm{Cdt} 2}$ and $\mathrm{SCF}^{\beta-\mathrm{TRCP}}$ as part of the degradation network to govern the timely destruction of Set8, and further suggest that aberrancies in these destruction pathways will likely cause adverse effects, leading to aberrant cell cycle progression or chromosome instability.

及-TRCP-mediated Set8 degradation affects cell cycle. It has been reported that both Set8 expression levels and H4K20me1 intensities fluctuate during the cell cycle, and Set8-mediated H4K20me plays a critical role in regulating cell cycle $e^{8,9,25}$. Consistent with a previous report ${ }^{9}$, we found that Dox-induced expression of Set8 ${ }^{\triangle \mathrm{PIP}}$ caused a prominent increase in G2/M cell populations compared with Set 8 WT (Fig. 6a and Supplementary Fig. 6a-h). Moreover, Set $8^{\mathrm{S} 253 \mathrm{~A}}$ also caused a robust G2/M cell cycle arrest compared with Set 8 WT. Interestingly, the Set $8^{\Delta \mathrm{PIP} / \mathrm{S} 253 \mathrm{~A}}$ mutant only led to a moderately increased $\mathrm{G} 2 / \mathrm{M}$ arrest compared with Set $8^{\Delta \mathrm{PIP}}$ (Fig. 6a). These results indicate that further investigation is required to explore the role of each of these degradation pathways in regulating Set8-mediated G2/M arrest.

Mechanistically, previous studies have revealed that ectopic expression of the Set 8 PIP mutant induced H4K20mel accumulation and phospho-Chk1 to cause the cell cycle defects 9 . To further determine the mechanism of $\beta$-TRCP-mediated degradation of Set 8 in regulation of cell cycle, we monitored the changes of pChk1, H4K20me1, H4K20me2, H4K20me3 and pSer10-H3 in cells ectopically expressing Set $8^{\mathrm{WT}}$, Set $8^{\Delta \mathrm{PIP}}$, Set $8^{\mathrm{S} 253 \mathrm{~A}}$ or $\mathrm{Set}^{\Delta \mathrm{PIP} / \mathrm{S} 253 \mathrm{~A}}$. Interestingly, we found that cells released from a late $\mathrm{G} 1 / \mathrm{S}$ phase arrest after hydroxyurea (HU) challenge, ectopic expression of WT-Set 8 only minimally affected cell cycle progression as it can be degraded in $\mathrm{G} 2 / \mathrm{M}$ phase and 
a

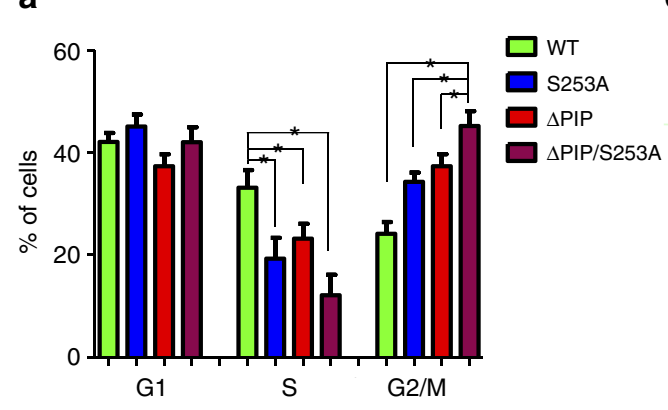

d

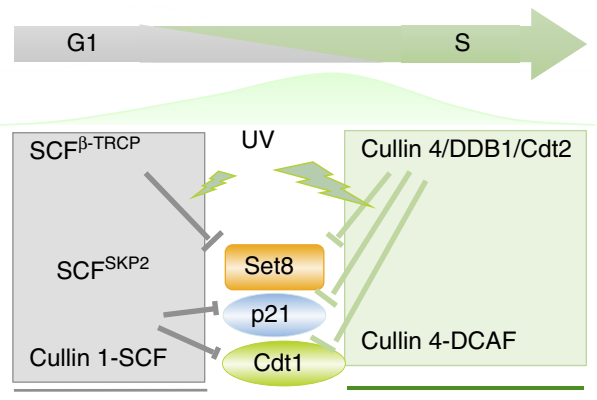

b
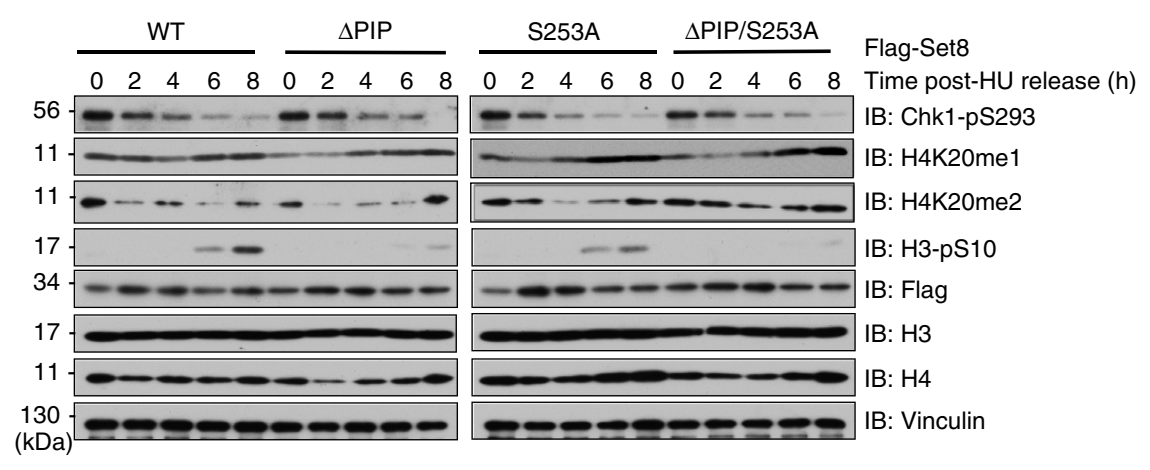

IB: Chk1-pS293

IB: H4K20me1

IB: H4K20me2

IB: H3-pS10

IB: Flag

IB: H3

IB: $\mathrm{H} 4$

IB: Vinculin

C

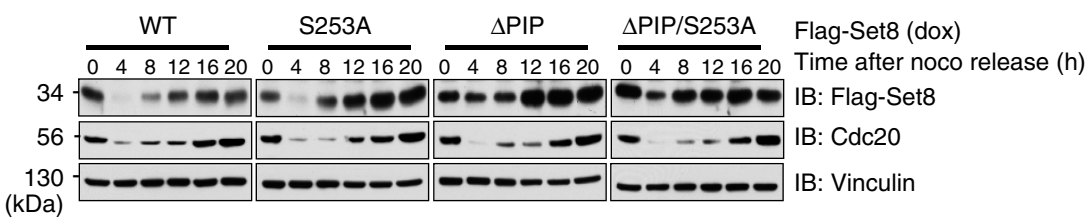

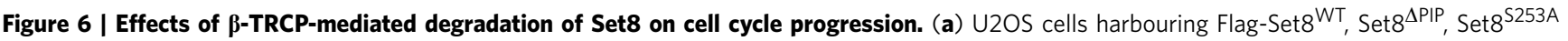
or Set $8^{\Delta \mathrm{PIP} / \mathrm{S} 253 \mathrm{~A}}$ were cultured in the presence of $0.1 \mu \mathrm{g} \mathrm{ml}{ }^{-1}$ doxycycline (Dox). Cell cycle was analysed by propidium iodide staining using fluorescenceactivated cell sorting. Data are shown as mean \pm s.d. of three independent experiments. ${ }^{\star} P<0.05$, Student's $t$-test. (b) U2OS cells harbouring inducible Flag-tagged Set8WT, Set8 $8^{\Delta \mathrm{PIP}}$, Set8 $8^{\mathrm{S} 253 \mathrm{~A}}$ or Set $8^{\mathrm{AIP} / \mathrm{S} 253 \mathrm{~A}}$ were synchronized in S phase with hydroxyurea (HU) for $24 \mathrm{~h}$. During the last $4 \mathrm{~h}$ in $\mathrm{HU}$, expression of Set8 was induced by Dox. Then, cells were released from HU in the presence of Dox, and IB analysis was performed to detect the indicated proteins. (c) U2OS cells harbouring inducible Flag-tagged Set8 ${ }^{\mathrm{WT}}$, Set $8^{\triangle \mathrm{PIP}}$, Set8 ${ }^{\mathrm{S} 253 \mathrm{~A}}$ or Set8 $8^{\triangle \mathrm{PIP} / \mathrm{S} 253 \mathrm{~A}}$ were synchronized in M phase with nocodazole for $18 \mathrm{~h}$. During the last $4 \mathrm{~h}$ in nocodazole, expression of Set8 was induced by Dox. Then, cells were released from nocodazole in the presence of Dox, and IB analysis was performed to detect the indicated proteins. (d) A schematic illustration of the signalling network that timely regulates the Set8 protein stability during the cell cycle progression.

cells enter $\mathrm{M}$ phase in around $6-8 \mathrm{~h}$ post-HU release, as evidenced by elevation of pSer10-H3. In keeping with previous reports 9 , expressing the more stabilized versions of Set $8^{\Delta \mathrm{PIP}}$ or Set $8^{\text {S253A }}$, led to a retardation of cell cycle progression, delaying cells to enter $M$ phase, as evidenced by reduced pSer10-H3 in 6$8 \mathrm{~h}$ time points. Furthermore, consistent with the robust potencies in arresting cells in G2/M phase, Dox-induced expression of the Set8 $8^{\Delta \mathrm{PIP} / \mathrm{S} 253 \mathrm{~A}}$ mutant led to a more dramatic delay for cells to enter $M$ phase, as there were only residual pSer10-H3 signals detected $8 \mathrm{~h}$ post-HU release (Fig. 6b). Consistent with an elevated methyltransferase activity, there were more H4K20me2 signals detected in the $8 \mathrm{~h}$ time point of Set $8^{\Delta \mathrm{PIP} / \mathrm{S} 253 \mathrm{~A}}$-expressing cells (Fig. 6b and Supplementary Fig. 6i,j). However, further studies are required to make the molecular connection between elevation of H4K20me 2 and increased activity of the p53/p21 tumor-suppressor pathway to functionally induce G2/M cell cycle arrest. Furthermore, in support of a critical role of both CRL4 ${ }^{\mathrm{Cdt} 2}$

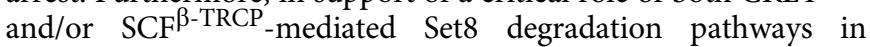
governing Set8 activity/abundance during the cell cycle progression, we observed that compared with WT-Set8, Set 8 S253A was more stable in the G1 phase ( $8 \mathrm{~h}$ post release), while the Set $8^{\triangle \mathrm{PIP}}$ was relatively more stable as cells enter into $\mathrm{S}$ phase (12-16 h post release) (Fig. 6c and Supplementary Fig. 6k).
Notably, the Set8 $8^{\Delta \mathrm{PIP} / \mathrm{S} 253 \mathrm{~A}}$ double mutant may evade both degradation pathways, thereby becoming stabilized in both G1 and $S$ phases (8-16 time points) (Fig. 6c). These results further support the notion that like many other cell cycle regulators including p21 and Cdt1 (refs 26,27), Set8 stability is likely controlled by different sets of E3 ligases including CRL4 ${ }^{\mathrm{Cdt} 2}$ and/or SCF ${ }^{\beta-T R C P}$ that may function in different cell cycle phases to timely control the abundance of Set8 (Fig. 6d).

\section{Discussion}

Previous studies have reported that $\mathrm{CRL}^{\mathrm{Cdt} 2}$ (refs 4,8,9) and $\mathrm{APC}^{\mathrm{Cdh} 1}$ (ref. 7) promote the degradation of Set8 during cell cycle, indicating that Set 8 is required to be maintained at precise levels by multiple E3 ligases for proper cell cycle progression. Here, we report SCF ${ }^{\beta-T R C P}$ as another E3 ligase that controls Set8 destruction to govern cell cycle transition. Different from other studies, we discovered CKI $\delta$ as the upstream kinase that phosphorylates Set8 at Ser253 to trigger its ubiquitination and subsequent degradation by $\mathrm{SCF}^{\beta-T R C P}$. In line with this finding, Set $8^{\mathrm{WT}}$, but not Set $8^{\mathrm{S} 253 \mathrm{~A}}$, interacted with $\beta$-TRCP. Moreover, Set ${ }^{\text {S253A }}$ displayed extended half-life in $\beta$-TRCP/CKI $\delta$-mediated degradation.Taken together, our data suggests a critical role for 
Ser253 phosphorylation in controlling Set8 stability. Since Set8 is an important cell cycle regulator, regulation of $\beta$-TRCP and CKI $\delta$ could govern proper cell cycle progression through control of Set8 stability. More importantly, as CKI $\delta$ has been reported by multiple groups to be activated by the DNA damage signals ${ }^{24,28,29}$, our results provide an important molecular link between DNA damage-induced kinase cascade and the timely destruction of Set8 (Supplementary Fig. 7).

It has been previously identified that CRL4 ${ }^{\mathrm{Cdt} 2}$ is responsible for Set8 proteolytic degradation in the $S$ phase of the cell cycle $8,12,30$. Specifically, CRL4 ${ }^{\text {Cdt2 }}$ promotes the degradation of Set8, which is dependent on the Set8-PCNA interaction, leading to attenuated levels of H4K20mel during S phase ${ }^{8}$. In S phase and after DNA damage, CRL4 ${ }^{\text {Cdt2 }}$ promotes the destruction of both Set8 and Cdt1 (ref. 9). The PIP degron of Set8 is required for this process. In support of this notion, we observed that Set $8^{\Delta P I P}$ induced greater G2/M arrest compared with Set 8 WT (Fig. 6a and Supplementary Fig. 6a-h). Similarly, Set $8^{\mathrm{S} 253 \mathrm{~A}}$ also caused a robust G2 arrest, indicating that $\beta$-TRCP-mediated Set8 degradation is also involved in regulating cell cycle progression. Multiple lines of evidence have indicated that inactivation of the CRL4 ${ }^{\text {Cdt2 }}$-mediated Set8 degradation inhibits cell proliferation ${ }^{8,9}$. In line with this, Set ${ }^{S 253 \AA}$, phenocopying Set $8^{\Delta \mathrm{PIP}}$, led to a marked inhibition in cell proliferation (Fig. $5 \mathrm{~d}$ ), suggesting that like CRL4 ${ }^{\text {Cdt2 }}, \beta$-TRCP-mediated Set 8 destruction also plays an essential role in cell proliferation. Furthermore, we obtained evidence from both biochemical and cellular assays showing that $\mathrm{CRL} 4^{\mathrm{Cdt} 2}$ and SCF ${ }^{\beta \text {-TRCP }}$ are likely independent pathways in governing Set8 stability. As such, simultaneous inactivation of both destruction mechanisms led to a more resistant mutant form of Set8 (Set8 $\left.8^{\Delta \mathrm{PIP} / \mathrm{S} 253 \mathrm{~A}}\right)$ that is more potent in inhibiting cell growth.

Recent studies have also highlighted the important role of Set8 in tumorigenesis. Specifically, Set8 expression is positively associated with metastasis in breast cancer patients ${ }^{31}$. Several studies have demonstrated that patients with low Set8 levels have longer survival in hepatocellular carcinoma ${ }^{32}$ and small-cell lung cancer $^{33}$. In addition, low expression of Set 8 contributes to a decreased risk of epithelial ovarian cancer ${ }^{34}$. Recently, Set8 was found to be involved in the androgen receptor (AR)-mediated transcription activation via interaction with $\mathrm{AR}$ and H4K20me1 in prostate cancer ${ }^{35}$. Overexpression of Set8 promoted AR-induced cell proliferation in prostate cancer cells ${ }^{35}$, suggesting that the function of Set8 in regulating cell proliferation remains largely controversial. More importantly, besides cell proliferation, Set8 was found to promote epithelialmesenchymal transition and invasion in breast cancer cells through interplay with Twist via its dual chromatin remodelling activity $^{31}$. Consistently, downregulation of Set8 by the tumour suppressor miRNA-7 inhibited H4K20me1, leading to suppression of epithelial-mesenchymal transition and metastasis of breast cancer cells, and sensitizing cells to DNA damages ${ }^{36}$. Altogether, these clinical studies suggest that Set8 largely functions as an oncogene, but might be primarily associated with late events such as cancer metastasis.

It is important to note that recent studies from multiple groups showed that acute expression of an oncogene such as Ha-Ras ${ }^{37}$, $\mathrm{Akt}^{38,39}, \mathrm{BRAF}^{40,41}$ or hypoxia-inducible factor 1 (HIF1) ${ }^{42}$, would rather lead to a growth arrest phenotype, but not accelerated cell proliferation. This feature is specifically termed OIS, in short for oncogene-induced senescence. In support of this notion, genetic deletion of phosphatase and tensin homolog (PTEN) deleted on chromosome 10 (refs 43,44) or von hippellindau (VHL) ${ }^{45}$ tumour suppressor also led to the onset of senescence, likely due to aberrant expression of pAkt and HIF oncoproteins, while ectopic expression of BRAF directly led to senescence in melanocytes ${ }^{40}$. We speculate that similarly, stabilization of the Set8 oncoprotein by disrupting either the CRL4 ${ }^{\mathrm{Cdt} 2}$ or the SCF ${ }^{\beta-T R C P}$ pathway might cause growth arrest (Figs $5 \mathrm{~d}$ and $6 \mathrm{a}-\mathrm{c}$ ) through the OIS mechanism. Given that $\beta$-TRCP-mediated degradation of Set8 is in a CKI $\delta$-dependent manner, modulation of $\beta$-TRCP and/or CKI $\delta$ may be beneficial in treating a variety of human cancers.

Collectively, our results provide mechanistic insights into a novel degradation pathway for Set8 during cell cycle transition. It is possible that like many other critical cell cycle regulators including p21 and Cdt2, the stability of Set 8 is tightly and synergistically regulated by multiple different E3 ligases (Fig. 6d). In $\mathrm{S}$ phase, Set8 destruction is primarily promoted by CRL4 ${ }^{\mathrm{Cdt} 2}$, while in G1 phase, Set 8 can be controlled by Skp 2 and $\beta$-TRCP in different cellular context, with the $\beta$-TRCP/CKI pathway devotes to DNA damage-induced Set8 destruction (Fig. 6d). Without a doubt, further investigations are required to decipher how the orchestration of three E3 ligases controls the timely degradation of Set8 to govern proper cell cycle progression. Given that Set8 is critically involved in cell cycle progression, our studies could provide a possible mechanism for dysregulated cell cycle in cancer and further suggest that targeting $\beta$-TRCP could be a promising approach for the treatment of human cancers.

\section{Methods}

Cell culture. 293T, HeLa, T98G and U2OS cells were obtained from the American Type Culture Collection (ATCC) and cultured in DMEM medium (Life Technologies, CA, USA) supplemented with $10 \%$ fetal bovine serum, penicillin and streptomycin. The cells were maintained in a $5 \% \mathrm{CO}_{2}$-humidified atmosphere at $37^{\circ} \mathrm{C}$. Cullin $4 A^{f l f l}$ (ref. 46) and Cullin $4 B^{f l / f l}$ (ref. 47) mouse embryonic fibroblasts were kindly provided and characterized by Dr Pengbo Zhou (Weill Cornell Medical College, New York, NY, USA).

Plasmids. Various Set8 mutants were generated using the QuikChange XI Site-Directed Mutagenesis Kit (Stratagene) according to the manufacturer's instructions. Human siRNA oligos against Skp2 (A-sense, 5'-AAGGUCUCUGG UGUUUGUAAG- $3^{\prime}$; B-sense, $5^{\prime}$-AAGCAUGUACAGGUGGCUGUU- $3^{\prime}$ ), $\beta$-TRCP $1+2$ (sense, $5^{\prime}$-AAGUGGAAUUUGUGGAACAUC- $3^{\prime}$ ) and Cdh1 (sense, $5^{\prime}$-UGAGAAGUCUCCCAGUCAGUU- $3^{\prime}$ ) were purchased from Dharmacon. Short hairpin RNAs (shRNA lentivirus vectors), including shRNA- $\beta$-TRCP1, shRNA- $\beta$-TRCP1 +2 , shRNA-GFP, Flag- $\beta$-TRCP1, Flag- $\beta$-TRCP1-R474A constructs, and CKI constructs were obtained from Dr Wade Harper (Harvard Medical School, Boston, MA, USA). shRNA lentiviral vectors against Cullin 1 were gifts from J. Wade Harper (Harvard Medical School). Myc-Cullin 1, Myc-Cullin 2, Myc-Cullin 3, Myc-Cullin 4 and Myc-Cullin 5 constructs were obtained from James DeCaprio (Dana-Farber Cancer Institute, Boston, MA, USA). Lentiviral shRNA constructs against green fluorescent protein (GFP) and various CKI isoforms were gifts from William Hahn (Dana-Farber Cancer Institute, Boston MA, USA). Lentiviral shRNA constructs against Cullin 4A and Cullin 4B were purchased from Open Biosystems (Lafayette, CO, USA).

Antibodies and reagents. Anti-Set8 (C18B7; $1: 1,000$ immunoblot (IB)), anti- $\beta$ catenin (D10A8; 1:1,000 IB), anti-Skp2 (D3G5; 1:1,000 IB), anti-Mcl-1 (D35A5; 1:1,000 IB), anti-G9A (C6H3; 1:1,000 IB), anti-Cullin 1 (4995; 1:1,000 IB) and anti- $\beta$-TRCP1 (4394; 1:1,000 IB) antibodies were purchased from Cell Signaling Technology. Anti-p27 (SC-528; 1:500 IB), anti-Cullin 1 (SC-17775; 1:1,000 IB), anti-casein kinase $1 \delta(\mathrm{H}-60 ; 1: 1,000 \mathrm{IB})$, anti-casein kinase $1 \alpha$ (SC-6474; 1:1,000 IB), anti-c-Myc (9E10; 1:1,000 IB, $2 \mu$ g immunoprecipitates (IP)) and polyclonal anti-HA antibodies (SC-805; 1:1,000 IB, $2 \mu \mathrm{g}$ IP) were purchased from Santa Cruz Biotechnology. Anti-Vinculin antibody (V-4505; 1:2,000 IB), monoclonal anti-Flag antibody (F-3165), polyclonal anti-Flag antibody (F-2425; 1:1,000 IB, $2 \mu \mathrm{g}$ IP), antiHA agarose beads (A-2095; 1:1,000 IB, $2 \mu \mathrm{g} \mathrm{IP}$ ), peroxidase-conjugated anti-mouse secondary antibody (A-4416; 1:3,000 IB) and peroxidase-conjugated anti-rabbit secondary antibody (A-4914; 1:3,000 IB) were purchased from Sigma. Monoclonal anti-HA antibody (MMS-101P) was purchased from Covance. Anti-GFP antibody (632380; 1:1,000 IB), anti-Cdh1 (34-2000; 1:1,000 IB) antibody, Oligofectamine, Lipofectamine and Plus reagents were purchased from Invitrogen. Anti-CUL 4B (16184-1-AP; 1:1,000 IB) antibody was purchased from Proteintech Group.

Immunoblots and immunoprecipitation. Cells were lysed in EBC lysis buffer (50 mM Tris $\mathrm{pH} 8.0,120 \mathrm{mM} \mathrm{NaCl}, 0.5 \% \mathrm{NP}-40$ ) supplemented with protease inhibitors (Roche) and phosphatase inhibitors (EMD Millipore). The protein concentrations were measured using the Bio-Rad protein assay reagent (Bio-Rad 
Laboratories, CA, USA). The lysates were then resolved by SDS-PAGE and immunoblotted with indicated antibodies as described before ${ }^{48}$. For immunoprecipitation assays, $800 \mu \mathrm{g}$ of protein lysates were incubated with the appropriate antibody $(1-2 \mu \mathrm{g})$ overnight at $4{ }^{\circ} \mathrm{C}$ followed by addition of carrier beads for $1 \mathrm{~h}$. Immunocomplexes were washed with NETN buffer $(20 \mathrm{mM}$ Tris, $\mathrm{pH}$ $8.0,100 \mathrm{mM} \mathrm{NaCl}, 1 \mathrm{mM}$ EDTA and $0.5 \% \mathrm{NP}-40$ ), then resolved by SDS-PAGE and immunoblotted with indicated antibodies. Quantification of the immunoblot band intensity was performed with ImageJ software. Full scans of IBs are presented in Supplementary Fig. 8.

Mass spectrometry analysis. Whole cellular extracts from Flag- $\beta$-TRCP1 stably expressing $293 \mathrm{~T}$ cells were subjected to affinity purification with anti-Flag antibody. The purified protein complex was resolved by SDS-PAGE and silver stained, and the bands were retrieved and analysed by mass spectrometry.

Real-time RT-PCR analysis. RNA was extracted using Qiagen RNeasy mini kit, and the reverse transcription reaction was performed using the ABI Taqman Reverse Transcriptional Reagents. After mixing the resulting template with $\beta$-TRCP2 (Hs00362667_m1) or GAPDH (Hs999999905_ml) primers and ABI Taqman Fast Universal PCR Master Mix, the real-time reverse transcription (RT)-PCR reaction was performed with the ABI-7500 Fast Real-time PCR system

Protein degradation analysis. Cells were transfected with a plasmid encoding a HA-tagged version of Set8 along with Flag- $\beta$-TRCP1, and a plasmid encoding GFP as an internal control, in the presence or absence of Myc-CKI $\delta$. After $40 \mathrm{~h}$, cells were lysed and subsequently immunoblot analysis was performed. For half-life studies, $20 \mu \mathrm{g} \mathrm{ml}^{-1}$ cycloheximide (CHX, Sigma) was added to the medium $40 \mathrm{~h}$ post transfection. At various time points thereafter, cells were lysed and immunoblot analysis was conducted to detect protein abundances.

In vitro ubiquitination assay. The in vitro ubiquitination assays were conducted. The SCF ${ }^{\beta-T R C P 1}$ (E3) complexes were purified from cells transfected with vectors encoding GST- $\beta$-TRCP1, Myc-Cul-1, Myc-Skp1 and HA-Rbx1. The indicated GST-Set8 (amino acids 173-267) proteins were incubated with purified, recombinant active CKI $\delta$ in the presence of ATP at $30^{\circ} \mathrm{C}$ for $30 \mathrm{~min}$. Then, the kinase reaction products were incubated with SCF ${ }^{\beta-T R C P 1}$ (E3) complexes in the presence of purified, recombinant active E1, E2, ATP, and ubiquitin at $30^{\circ} \mathrm{C}$ for $45 \mathrm{~min}$. The immunoblot analysis was performed using the indicated antibodies.

Cell transfection. For cell transfection, cells were seeded in $60 \mathrm{~mm}$ plates and transfected using Lipofectamine 2000 (Invitrogen) in OptiMEM medium (Invitrogen) for $48 \mathrm{~h}$ according to the manufacturer's instructions. Real-time RT-PCR or western blot analysis was used to detect the efficacy of transfection.

Cell cycle and colony formation and soft agar assay. Cells were collected, fixed with $75 \%$ ethanol and stained with propidium iodide for flow cytometry analysis to assess cell cycle. Cells were seeded into the gelatinized plates. After 4 days, the colonies were stained on the plates with crystal violet and counted. The numbers of surviving colonies were calculated as the average of triplicates. Two percent melting point agar was prepared and mixed with RPMI1640 to make the 0.4 and $0.8 \%$ agar in $50^{\circ} \mathrm{C}$. Two millilitre $0.8 \%$ agar was added in the bottom of the six-well plate. About $3 \times 10^{4}$ cells and $2 \mathrm{ml} 0.4 \%$ agar were mixed, and the mixture was added on the top of $0.8 \%$ agar. After routine culture for 2 weeks, colony numbers were counted under a microscope.

Statistical analysis. The significance of the data between experimental groups was determined by Student's $t$-test. $P<0.05$ was considered to be significant.

\section{References}

1. Beck, D. B., Oda, H., Shen, S. S. \& Reinberg, D. PR-Set7 and H4K20me1: at the crossroads of genome integrity, cell cycle, chromosome condensation, and transcription. Genes Dev. 26, 325-337 (2012).

2. Jorgensen, S., Schotta, G. \& Sorensen, C. S. Histone H4 lysine 20 methylation: key player in epigenetic regulation of genomic integrity. Nucleic Acids Res. 41, 2797-2806 (2013).

3. Schotta, G. et al. A chromatin-wide transition to $\mathrm{H} 4 \mathrm{~K} 20$ monomethylation impairs genome integrity and programmed DNA rearrangements in the mouse. Genes Dev. 22, 2048-2061 (2008).

4. Oda, H. et al. Regulation of the histone H4 monomethylase PR-Set7 by CRL4(Cdt2)-mediated PCNA-dependent degradation during DNA damage. Mol. Cell 40, 364-376 (2010).

5. Shi, X. et al. Modulation of p53 function by SET8-mediated methylation at lysine 382. Mol. Cell 27, 636-646 (2007).

6. Wakabayashi, K. et al. The peroxisome proliferator-activated receptor gamma/ retinoid $\mathrm{X}$ receptor alpha heterodimer targets the histone modification enzyme
PR-Set7/Setd8 gene and regulates adipogenesis through a positive feedback loop. Mol. Cell. Biol. 29, 3544-3555 (2009).

7. Wu, S. et al. Dynamic regulation of the PR-Set7 histone methyltransferase is required for normal cell cycle progression. Genes Dev. 24, 2531-2542 (2010).

8. Abbas, T. et al. CRL4(Cdt2) regulates cell proliferation and histone gene expression by targeting PR-Set7/Set8 for degradation. Mol. Cell 40, 9-21 (2010).

9. Centore, R. C. et al. CRL4(Cdt2)-mediated destruction of the histone methyltransferase Set8 prevents premature chromatin compaction in S phase. Mol. Cell 40, 22-33 (2010).

10. Yin, Y., Yu, V. C., Zhu, G. \& Chang, D. C. SET8 plays a role in controlling G1/S transition by blocking lysine acetylation in histone through binding to $\mathrm{H} 4$ N-terminal tail. Cell Cycle 7, 1423-1432 (2008).

11. Yan, Q. et al. BBAP monoubiquitylates histone $\mathrm{H} 4$ at lysine 91 and selectively modulates the DNA damage response. Mol. Cell 36, 110-120 (2009).

12. Jorgensen, S. et al. SET8 is degraded via PCNA-coupled CRL4(CDT2) ubiquitylation in S phase and after UV irradiation. J. Cell Biol. 192, 43-54 (2011).

13. Frescas, D. \& Pagano, M. Deregulated proteolysis by the F-box proteins SKP2 and beta-TrCP: tipping the scales of cancer. Nat. Rev. Cancer. 8, 438-449 (2008).

14. Guardavaccaro, D. et al. Control of meiotic and mitotic progression by the F box protein beta-Trcp1 in vivo. Dev. Cell 4, 799-812 (2003).

15. Margottin-Goguet, F. et al. Prophase destruction of Emil by the SCF(betaTrCP/Slimb) ubiquitin ligase activates the anaphase promoting complex to allow progression beyond prometaphase. Dev. Cell 4, 813-826 (2003).

16. Watanabe, N. et al. M-phase kinases induce phospho-dependent ubiquitination of somatic Weel by SCFbeta-TrCP. Proc. Natl Acad. Sci. USA 101, 4419-4424 (2004).

17. Jin, J. et al. SCFbeta-TRCP links Chk1 signaling to degradation of the Cdc25A protein phosphatase. Genes Dev. 17, 3062-3074 (2003).

18. Busino, L. et al. Degradation of Cdc25A by beta-TrCP during $S$ phase and in response to DNA damage. Nature 426, 87-91 (2003).

19. Shaik, S. et al. SCF(beta-TRCP) suppresses angiogenesis and thyroid cancer cell migration by promoting ubiquitination and destruction of VEGF receptor 2. J. Exp. Med. 209, 1289-1307 (2012).

20. Gao, D. et al. Cdh1 regulates cell cycle through modulating the claspin/Chk1 and the Rb/E2F1 pathways. Mol. Biol. Cell. 20, 3305-3316 (2009).

21. Gao, D. et al. mTOR drives its own activation via SCF(betaTrCP)-dependent degradation of the mTOR inhibitor DEPTOR. Mol. Cell 44, 290-303 (2011).

22. Huen, M. S., Sy, S. M., van Deursen, J. M. \& Chen, J. Direct interaction between SET8 and proliferating cell nuclear antigen couples H4-K20 methylation with DNA replication. J. Biol. Chem. 283, 11073-11077 (2008).

23. Wang, Z., Liu, P., Inuzuka, H. \& Wei, W. Roles of F-box proteins in cancer. Nat. Rev. Cancer 14, 233-247 (2014).

24. Inuzuka, H. et al. Phosphorylation by casein kinase I promotes the turnover of the Mdm2 oncoprotein via the SCF(beta-TRCP) ubiquitin ligase. Cancer Cell 18, 147-159 (2010).

25. Lee, J. \& Zhou, P. SETting the clock for histone H4 monomethylation. Mol. Cell 40, 345-346 (2010).

26. Shibata, E., Abbas, T., Huang, X., Wohlschlegel, J. A. \& Dutta, A. Selective ubiquitylation of p21 and Cdt1 by UBCH8 and UBE2G ubiquitin-conjugating enzymes via the CRL4Cdt2 ubiquitin ligase complex. Mol. Cell. Biol. 31, 3136-3145 (2011).

27. Abbas, T. et al. PCNA-dependent regulation of p21 ubiquitylation and degradation via the CRL4Cdt2 ubiquitin ligase complex. Genes Dev. 22, 2496-2506 (2008).

28. Lim, S. \& Kaldis, P. Cdks, cyclins and CKIs: roles beyond cell cycle regulation. Development 140, 3079-3093 (2013).

29. Wang, Z. et al. DNA damage-induced activation of ATM promotes beta-TRCPmediated Mdm2 ubiquitination and destruction. Oncotarget 3, 1026-1035 (2012).

30. Tardat, M. et al. The histone H4 Lys 20 methyltransferase PR-Set7 regulates replication origins in mammalian cells. Nat. Cell. Biol. 12, 1086-1093 (2010).

31. Yang, F. et al. SET8 promotes epithelial-mesenchymal transition and confers TWIST dual transcriptional activities. EMBO J. 31, 110-123 (2012).

32. Guo, Z. et al. A polymorphism at the miR-502 binding site in the 3 '-untranslated region of the histone methyltransferase SET8 is associated with hepatocellular carcinoma outcome. Int. J. Cancer 131, 1318-1322 (2012).

33. Ding, C., Li, R., Peng, J., Li, S. \& Guo, Z. A polymorphism at the miR-502 binding site in the 3' untranslated region of the SET8 gene is associated with the outcome of small-cell lung cancer. Exp. Ther. Med. 3, 689-692 (2012).

34. Wang, C., Guo, Z., Wu, C., Li, Y. \& Kang, S. A polymorphism at the miR-502 binding site in the 3' untranslated region of the SET8 gene is associated with the risk of epithelial ovarian cancer. Cancer Genet. 205, 373-376 (2012).

35. Yao, L. et al. Histone H4 Lys 20 methyltransferase SET8 promotes androgen receptor-mediated transcription activation in prostate cancer. Biochem. Biophys. Res. Commun. 18, 692-696 (2014). 
36. Yu, N. et al. microRNA-7 suppresses the invasive potential of breast cancer cells and sensitizes cells to DNA damages by targeting histone methyltransferase SET8. J. Biol. Chem. 288, 19633-19642 (2013).

37. Serrano, M., Lin, A. W., McCurrach, M. E., Beach, D. \& Lowe, S. W. Oncogenic ras provokes premature cell senescence associated with accumulation of p53 and p16INK4a. Cell 88, 593-602 (1997).

38. Xu, Y., Li, N., Xiang, R. \& Sun, P. Emerging roles of the p38 MAPK and $\mathrm{PI} 3 \mathrm{~K} / \mathrm{AKT} / \mathrm{mTOR}$ pathways in oncogene-induced senescence. Trends Biochem. Sci. 39, 268-276 (2014).

39. Astle, M. V. et al. AKT induces senescence in human cells via mTORC1 and p53 in the absence of DNA damage: implications for targeting mTOR during malignancy. Oncogene 31, 1949-1962 (2012).

40. Cao, J. et al. MC1R is a potent regulator of PTEN after UV exposure in melanocytes. Mol. Cell 51, 409-422 (2013).

41. Vredeveld, L. C. et al. Abrogation of BRAFV600E-induced senescence by PI3K pathway activation contributes to melanomagenesis. Genes Dev. 26, 1055-1069 (2012).

42. Kilic Eren, M. \& Tabor, V. The role of hypoxia inducible factor-1 alpha in bypassing oncogene-induced senescence. PLoS ONE 9, e101064 (2014).

43. Chen, Z. et al. Crucial role of p53-dependent cellular senescence in suppression of Pten-deficient tumorigenesis. Nature 436, 725-730 (2005).

44. Nardella, C. et al. Aberrant Rheb-mediated mTORC1 activation and Pten haploinsufficiency are cooperative oncogenic events. Genes Dev. 22, 2172-2177 (2008).

45. Young, A. P. et al. VHL loss actuates a HIF-independent senescence programme mediated by Rb and p400. Nat. Cell. Biol. 10, 361-369 (2008).

46. Liu, L. et al. CUL4A abrogation augments DNA damage response and protection against skin carcinogenesis. Mol. Cell 34, 451-460 (2009).

47. Liu, L. et al. Essential role of the CUL4B ubiquitin ligase in extra-embryonic tissue development during mouse embryogenesis. Cell Res. 22, 1258-1269 (2012).

48. Inuzuka, H. et al. Acetylation-dependent regulation of Skp2 function. Cell 150, 179-193 (2012).

\section{Acknowledgements}

This work was supported in part by the NIH grants to W.W. (GM094777 and CA177910). W.W. is an ACS research scholar and a LLS research scholar. This work was also supported by grant from NSFC $(81172087,81572936)$ and the priority academic program development of Jiangsu higher education institutions. H.I. is supported by AG041218 and X.D. is supported by NIH T32 training grant 5T32HL007893-18.

\section{Author contributions}

Z.W. and W.W. designed the study. Z.W., X.D., J.Z., H.I., L.Wan, X.L., L.Wang, X.Y., and D.G. performed and analysed the biological experiments. L.S. and L.Z. helped with the discussion. Z.W. and W.W. wrote the manuscript. W.W. guided all of the aspects of this study.

\section{Additional information}

Supplementary Information accompanies this paper at http://www.nature.com/ naturecommunications

Competing financial interests: The authors declare no competing financial interests.

Reprints and permission information is available online at http://npg.nature.com/ reprintsandpermissions/

How to cite this article: Wang, Z. et al. $\mathrm{SCF}^{\beta-T R C P}$ promotes cell growth by targeting PR-Set7/Set8 for degradation. Nat. Commun. 6:10185 doi: 10.1038/ncomms10185 (2015).

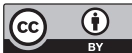

This work is licensed under a Creative Commons Attribution 4.0 International License. The images or other third party material in this article are included in the article's Creative Commons license, unless indicated otherwise in the credit line; if the material is not included under the Creative Commons license, users will need to obtain permission from the license holder to reproduce the material To view a copy of this license, visit http://creativecommons.org/licenses/by/4.0/ 